Journal of Al-Azhar University Engineering Sector

Vol.15, No. 55, April, 2020, 444-458

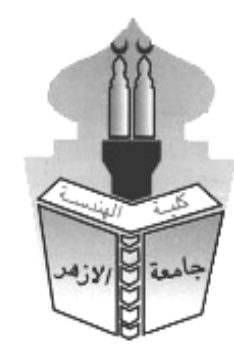

\title{
NUMERICAL SIMULATION OF PERMEABLE CAISSONS BREAKWATERS
}

\author{
Mohamed Ibrahim Mohamed \\ Civil Engineering Department, Faculty of Engineering, Al-Azhar University, Cairo, Egypt. \\ E-mail: anasamer3337@ azhar.edu.eg
}

\begin{abstract}
This research shows a numerical simulation study by (flow-3d) numerical model of a wave's barrier, which is precast concrete caissons suspended on spaced piles of suspension and partly submerged in water. The results of the numerical model have been validated by comparing its results with previous laboratory results for the same barrier this research shows that there is a great agreement between the results of the current numerical model and the previous laboratory model. The current numerical model presented values of wave transmission factors lower than other experimental models presented by other authors for cases of convergent wave barriers from this study and the study showed a decrease Wave transmission factor (kt) with increasing relative wave barrier width (B / h), where (h) water depth and (B) barrier width are also decreasing with increasing relative wave barrier count (D / h) where (D) the barrier draft. The efficiency of these barriers increases as the waves affecting them are relatively short. The effect of the sea floor slope increases on the efficiency of the proposed breakwater. The transmission coefficient (kt) decreases with increasing sea-floor slope. For short waves, the wave barrier is very effective in reducing the energy of the transverse wave, regardless of structural arrangements and sea floor conditions. The wave vortices and velocities increase around the barrier as the wave period (T) decreases.
\end{abstract}

KEYWORDS: Numerical, permeable, Pile System, Vortex, Caissons

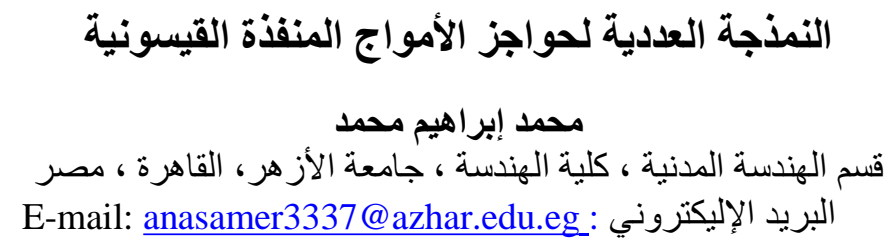

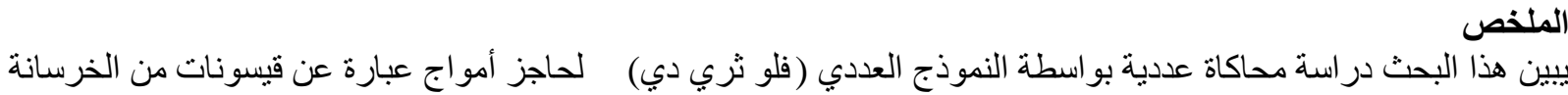

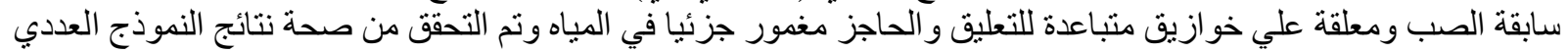

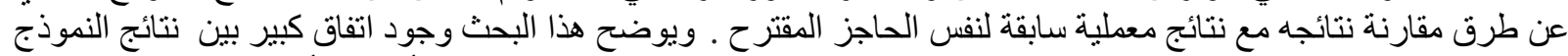

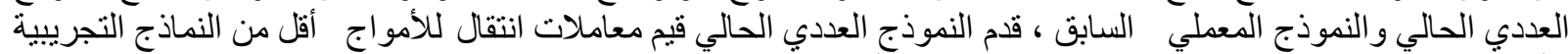

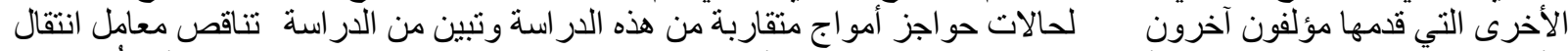

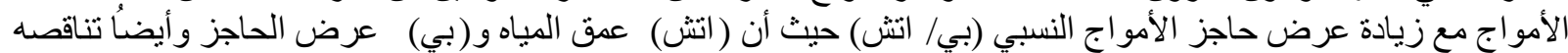

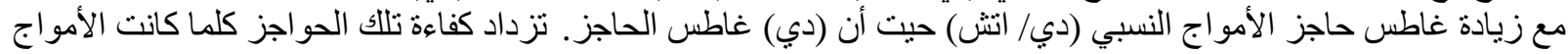




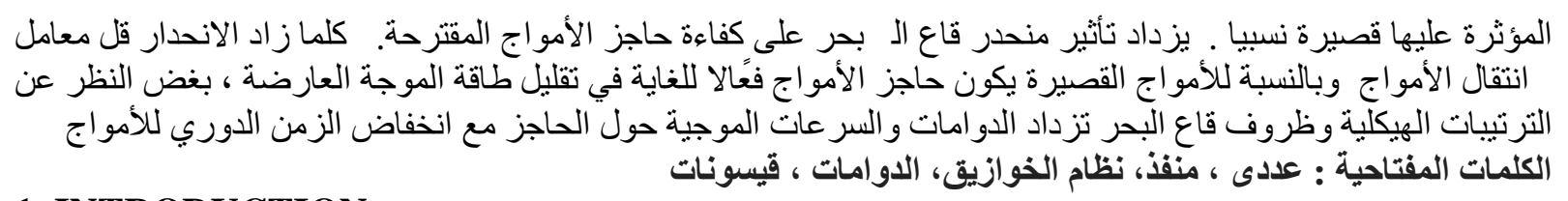

\section{INTRODUCTION}

Coastal engineering is a branch of civil engineering that deals with coastal problems, suggest ideas, and propose solutions. Problems which are commonly encountered in such environment and which directly affect the coasts are; the shore line stabilization, back shore protection, inlet stabilization, and harbor or marina protection. Shore protection and beach stabilization are important topics because development and use of coastal regions for recreational and commercial activities plays an increasing role in the national income in many countries around the world, especially Egypt. The Egyptian government has focused on developing the Red sea and the Mediterranean Sea coasts. The Egyptian government went ahead in developing and constructing seaside resorts in addition to a series of recreational harbors and villages to improve the tourism facilities. A major problem for coastal developing is protecting the largest possible shore area at the lowest possible cost. In other words, choosing and implementing the most economic shore structure protection type. There are many types of coastal protection structures such as; breakwaters, seawalls, groins, jetties, and artificial headlands. In addition, artificial beach nourishment is an alternative method of coastal protection. Breakwaters are used along shorelines, channel entrances, beaches, harbors, or marinas, and are considered a suitable coastal protection structures for many shore protection applications. Breakwaters may vary in type according to their intended use. The main breakwater types are; rubble mound breakwaters, massive vertical face breakwaters (block type, caisson type, cellular type), flexible breakwaters (row of piles, sheet piles), and Partial protection breakwaters (floating type, submerged type, detached type, screen types).

\subsection{Problem Statement}

From the partial protection breakwaters, there are one type that can be proposed for protecting coasts and recreational harbors. The partially immersed fixed body is the breakwater model which consists of a caisson partially immersed in the seawater and supported on a large spaced pile system. This breakwater type may be used because of the following:

1. Used as one of the most conventional solution in deeper waters.

2. Used in the coastal sites of low soil bearing capacity.

3. Successfully employed for low and moderate wave energy applications.

4. Allows for continuous refreshing the shore area water masses which in turn minimizes the pollution aspects, giving great advantage of such type of breakwaters over that acts like barrier walls.

5. Occupy small zone so that, not affecting the seabed creatures.

\subsection{The Research Objectives}

The objectives of this study are:

1. To propose suitable breakwater types for protecting coasts and recreational harbors lacking into consideration both efficiency and cost

2. To get the efficiency of the proposed breakwater type numerically under various prevailing environmental conditions.

\subsection{Research Methodology}

To fulfill the above-mentioned objectives, the following approaches are adapted:

1. One breakwater type is proposed:

a- Caisson type supported on large spaced pile system.

2. The numerical models, applying the finite volume theory to solve the three-dimensional Reynolds- Averaged Navier -Stokes (RANS) equations. Using "Computational Fluid Dynamics" 
(CFD) code FLOW-3D is developed to get the efficiency of the caisson supported on large spaced pile system.

3.Series of numerical study are carried out on the caisson supported on large space file system to check the validity of the experimental model.

The proposed models were studied in a previous laboratory experimental previous study of (Koraim, A.S., 2005). [14] "Suggested Model for the Protection of Shores and Marina"

\section{LITERATURE REVIEW}

When water waves strike a breakwater, the incident wave energy being reflected, some energy dissipated through the breakwater, and the remaining energy being transmitted behind that obstacle. Breakwaters are used along shoreline, channel entrances, beaches, harbors, or marinas may vary in type according to the required degree of wave protection and economics. The primary function of a breakwater is to provide shore protection by controlling the wave height allowed to be transmitted along the coasts. The breakwaters can be classified according to the degree of protection from the waves and from these types, Semi-submersible barriers. This breakwater system consists of an array of individual floating geometric forms, small relative to the wave length, anchored to the seabed, but not tied to each other.

The efficiency of the semi-immersed box breakwater was experimentally and theoretically studied by many researchers. Macagno (1953) [1], Stoker (1957) [3], Carver (1979) [3] and Reddy and Neelamanit (1992) [4] developed a theoretical solution for the partial transmission and reflection of gravity waves. Ito and Chiba (1972) [5] and Adee and Martin (1974) [6] studied the floating breakwater of arbitrary shape numerically. Also, Sutko and Haden (1974) [7], Fugazza and Natale (1988) [8] studied experimentally the efficiency of the floating breakwaters. Tolba (1998) [9] Summarized the performance of the rectangular floating breakwaters theoretically and experimentally. The tested models were fixed model, limited roll motion model, heave motion model, and floating model connected with vertical plate. Experiments were performed in Bergische University, Germany in a wave flume $24.0 \mathrm{~m}$ long, $0.50 \mathrm{~m}$ deep and $0.30 \mathrm{~m}$ wide. The effect of the breakwater heave motion on the breakwater performance was also analyzed theoretically. The transmission and reflection coefficients were determined for the different models, the height of the heave motion and the wave pressure on the breakwater surfaces was also determined. It was found that the transmission coefficient decreases and the reflection coefficient increases as the breakwater draft or width increases. For the breakwater moving only in heave motion, the results show that, the higher breakwater draft gave higher reflection and lower transmission. Heikal, (2004) [10] experimentally examined the wave pattern and seabed scour around rectangular semi-immersed bodies supported on piles in a sandy movable bed. Several experiments were conducted in a tilting wave flume $12 \mathrm{~m}$ long, $0.45 \mathrm{~m}$ deep and $0.30 \mathrm{~m}$ wide, at the Hydraulics Laboratory of Zagazig University. The proposed structure efficiency was measured at different locations along the wave flume. The seabed scours and deposition was also measured around the breakwater model and locally at the supporting piles. The efficiency of the proposed breakwater system was found to be remarkable when the relative submergence of the body increased, especially under short waves. The seabed scours locally increased around the piles for intermediate waves when the relative body draft decreased. Ioanna et al (2009) [11] investigated numerically the performance of an array of multiple pile-restrained floating breakwaters connected by hinges under the action of monochromatic incident waves in the frequency domain. The performance of the various configurations of multiple floating breakwaters examined is compared with the performance of a single pile-restrained breakwater with no hinges. The increase of the relative width $(\mathrm{B} / \mathrm{L})$ leads to reduction of $(\mathrm{Kt})$ and therefore, improvement of the effectiveness, especially for $\mathrm{B} / \mathrm{L} \geq 0.8$. This is attributed to low amplitudes of the diffracted waves and to low response level that results in reduced radiation waves. Wang and Sun (2010) [12] investigated experimentally a floating breakwater fabricated with large numbers of diamond-shaped blocks. Four sorts (with equal number of blocks) of geometrical configurations had been involved in the contrasting analyses. Various factors including water 
depth, relative width of the floating body. Influence of geometrical configuration of the moored floating breakwater on transmission coefficient $(\mathrm{kt})$. The results showed that the relative width played a crucial role in the performance of wave attenuation, and the values of kt was decreased markedly as B/L was increased in each case examined. Rageh and Koraim (2010) [13] investigated experimentally the performance of a breakwater, which consists of caissons supporting on the two or three rows of piles. The efficiency of the breakwater was presented as a function of the transmission, the reflection and the wave energy loss coefficients. Different characteristics of the caisson structure and the supporting pile system were tested. It was found that, the proposed breakwater helps in dissipating about 10 to $30 \%$ from the incident wave energy. Also, when the relative breakwater draft $(\mathrm{kD})$ more than 0.8 ; the efficiency of the proposed breakwater becomes high in decreasing the transmitted waves and the reflection coefficient not affected by the increasing or decreasing the breakwater width or pile characteristics. Koraim, A.S., 2005 [14] investigated experimentally the performance of the caisson supported on a large spaced pile system and pile breakwaters consisted of one row of circular and square piles to check the validity of the proposed two theoretical models. In addition the caisson supported on a closely spaced pile system and pile breakwaters consisted of two rows of circular and square piles arranged in straight and staggered positions were also studied. Additionally, the effect of the three breakwater types on the scour at the breakwater zone was investigated to determine the maximum scour and deposition. . Ahmed 2014[15] investigated regular wave interaction using a numerical model of (FLOW-3D, VOF) with a single vertical perforated wall. Mohamed 2018, [16] studied the efficiency hydrodynamic performance of double half pipes barriers was investigated, physically and numerically by Flow-3D. In this study, the efficiency of the proposed breakwaters is investigated using numerical models. The breakwaters efficiency was measured as function of transmission, reflection, and wave energy dissipation coefficients.

The previous researchers investigated pile, slotted and floating breakwaters using several models and several methods. In this study, based on the shortcomings of the previous Studies the efficiency of one breakwater type was studied using numerical model (FLOW-3D) and validated experimentally in previous study, for determining the effect of the purposed breakwater on wave's parameters. The characteristics of the barriers proposed numerically were also studied by a program (FLOW-3D).

\section{3- DESCRIPTION OF THE EXPERIMENTAL MODEL ACCORDING TO THE PREVIOUS EXPERIMENTAL STUDY (KORAIM 2005)}

The sequence of the experimental tests was presented as follows according to the previous laboratory study Koraim, A.S., 2005 [15]. One breakwater type is proposed as following.

\subsection{The breakwater a caisson supported on a Large Spaced Pile System}

1. The wave flume was filled with water to the required depth and the velocity of wave generator was adjusted to give the required wave periods $(\mathrm{T}=0.68,1.0,1.57$ and $3.0 \mathrm{sec}$.) and the incident wave heights $(\mathrm{Hi})$ were recorded.

2. The model of $(\mathrm{D}=0.0, \mathrm{~B}=1.0 \mathrm{~cm})$ was fixed at the bed and the bed slope was adjusted to $\mathrm{sb}=0 \%$.

3. The velocity of wave generator was adjusted to give the required initial wave period $(\mathrm{T}=$ $0.68 \mathrm{sec}$.).

4. The wave generator was operated to reach the steady wave condition and the wave height shoreward (Ht) was recorded by using the Digital Ultrasonic Water Surface Profile Instrument as follows:

$$
\mathrm{Ht}=\text { the maximum reading }- \text { The minimum reading }
$$

$\mathrm{kt}=\mathrm{Ht} / \mathrm{Hi}$

1. The velocity of the wave generator was changed to give different wave periods ( $\mathrm{T}=1.0$, 1.57 and $3.0 \mathrm{sec}$.) and the wave heights were recorded. 
2. The previous steps were repeated for different breakwater drafts $(\mathrm{D}=4,8,12,16 \mathrm{~cm})$ and the wave heights were recorded.

3. The previous steps were repeated for different breakwater models $(B=10,20,30$, and 40 $\mathrm{cm}$ ) and the wave heights were recorded. As in table (1) and fig. (1) \& (2)

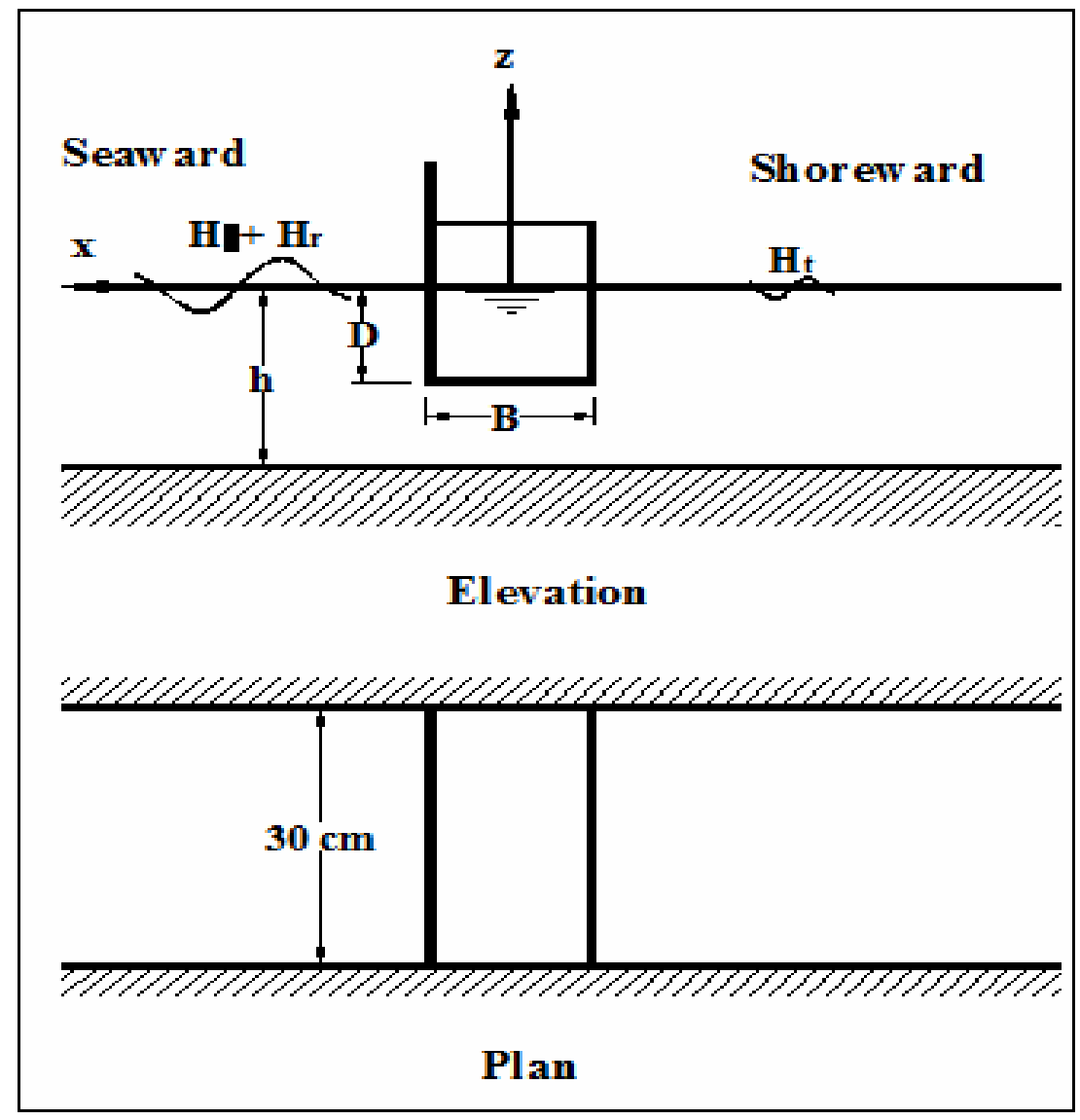

Fig. (1) The Details Of The Caisson Supported On Large Spaced Pile System

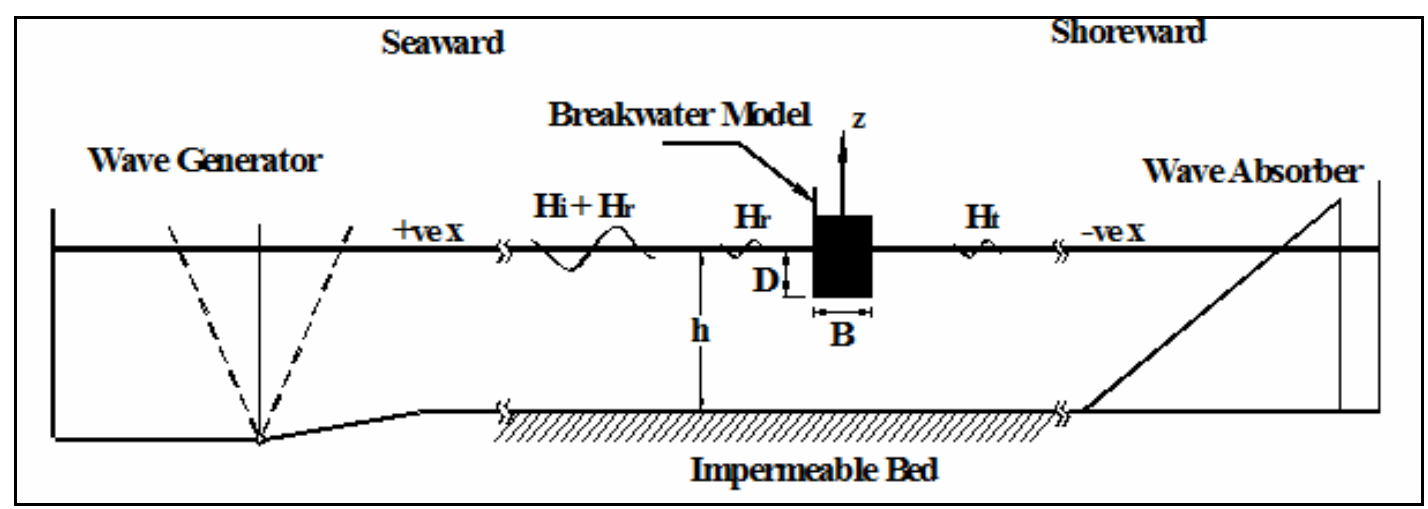

Fig. (2) Definition Sketch For Breakwater Model In The Wave Flume 
Table (1) The Previous Experimental Model Setup Parameters For The Caisson Supported On Large Spaced Pile System.

\begin{tabular}{|l|l|l|}
\hline Parameter & The ranges & Unites \\
\hline Water Depth (h) & 20 & $(\mathrm{~cm})$ \\
\hline Wave Periods (T) & $0.68,1.0,1.57$ and 3.0 & $(\mathrm{sec})$. \\
\hline Wave Length (L) & 65 to 490 & $(\mathrm{~cm})$ \\
\hline Breakwater Width (B) & $1,10,20,30$ and 40 & $(\mathrm{~cm})$ \\
\hline Breakwater Draft (D) & $0,4,8,12$ and 16 & $(\mathrm{~cm})$ \\
\hline
\end{tabular}

\section{VERIFICATION OF NUMERICAL MODEL ANALYSIS}

This section is concerned with presenting and analyzing the numerical results obtained by computer programs "Computational Fluid Dynamics" (CFD) code FLOW-3Dare used to determine the efficiency of the caisson on large spaced piles and the pile breakwaters. The validity of the above-mentioned numerical models in predicting the breakwaters efficiency is also verified by comparing their results with the present experimental results along with other experimental and theoretical results obtained from other studies.

\subsection{Verification Of Numerical Model Results With Previous Experimental Study}

Figure (3) shows a comparison between the present numerical model and experimental transmission coefficients for different $\mathrm{kh}$ for $\mathrm{B} / \mathrm{h}$ is equal to 1.5. The figure shows a reasonable agreement between the theoretical and experimental results for $\mathrm{kh}=1.83$, Figure (3a). In addition, the numerical model underestimates the transmission coefficient $(\mathrm{kt})$ by about $5 \%$ for $\mathrm{kh}=1.04$, Figure ( $3 b)$. On the other hand, a reasonable agreement between the numerical model and experimental results for $k h=0.31$, Figure (3c), especially when $D / h \geq 0.4$. Figure (4) shows comparisons between the present numerical model and the experimental transmission coefficients for different wave and structural conditions. The Figure shows comparison between the present numerical and experimental transmission coefficients for different kh when $\mathrm{B} / \mathrm{h}=1$. The figure shows a reasonable agreement between the numerical and experimental results when $\mathrm{kh} \geq 1.04$, as shown in Figures (4a) and (4b). On the other hand, the numerical model overestimates the transmission coefficient (kt) by about 5 to $10 \%$ for $\mathrm{kh}<1.04$, Figures $(4 \mathrm{c})$ and $(4 \mathrm{~d})$, especially when $\mathrm{D} / \mathrm{h}<0.6$, after which the numerical estimation become more accurate. Figure (5) shows a comparison between the present numerical model transmission and reflection coefficients, [FLOW-3D], and those introduced by [Koraim (2005)], are presented in Figure (5), for different relative drafts $(\mathrm{D} / \mathrm{h})$ when the relative breakwater width $(\mathrm{B} / \mathrm{h})$ is equal to 0.5 . The figure shows a reasonable agreement between the numerical and the experimental transmission coefficients especially when $\mathrm{kh}>0.8$, while the numerical model overestimates kt by about 5 to $20 \%$ when $\mathrm{kh}$ $<0.8$. The figure also shows an acceptable agreement between the numerical and experimental reflection coefficients, especially when $\mathrm{kh}>2.0$, while the numerical model overestimates $\mathrm{kr}$ by about 5 to $10 \%$, when $\mathrm{kh} \geq 2.0$. 
NUMERICAL SIMULATION OF PERMEABLE CAISSONS BREAKWATERS
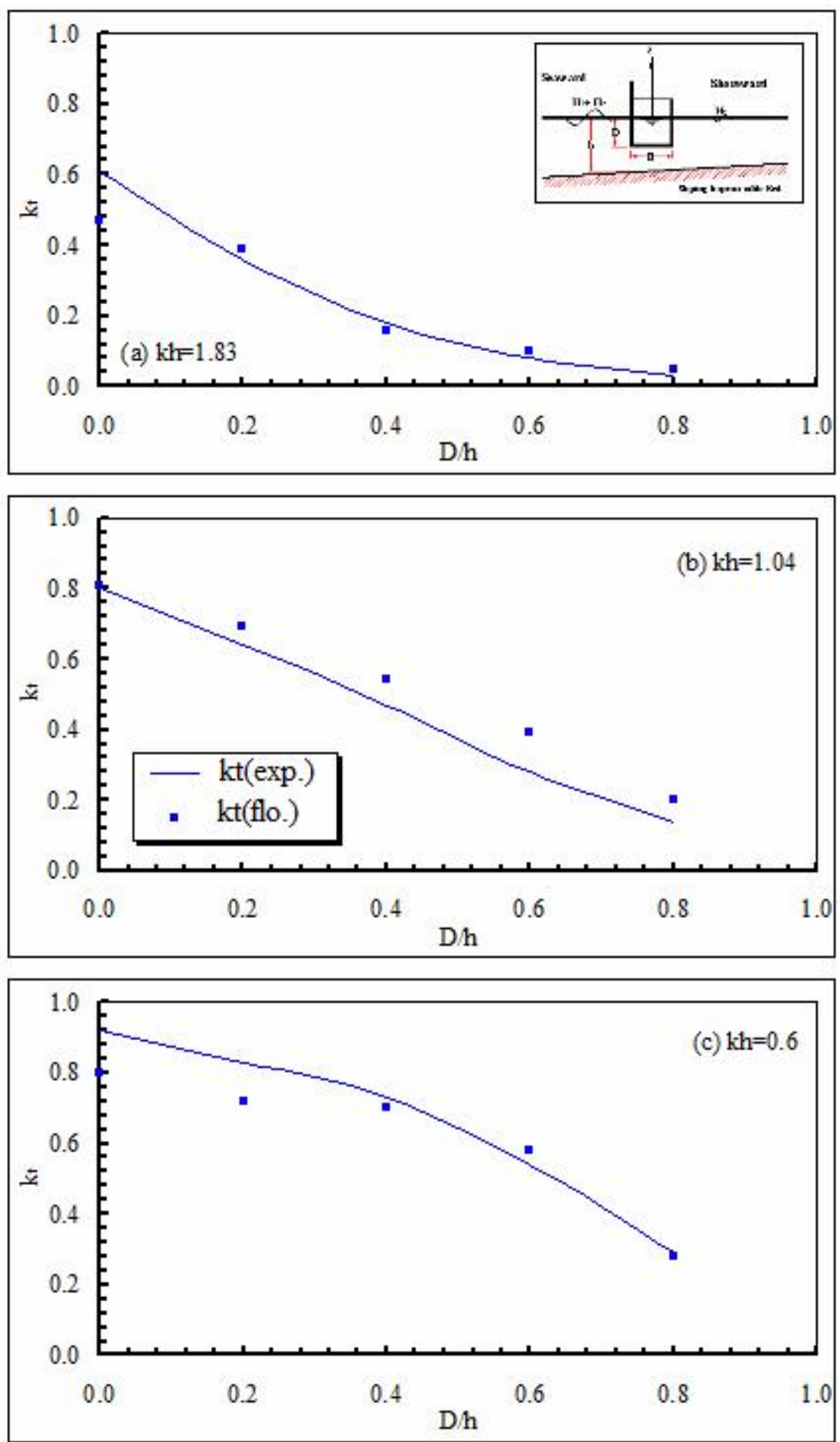

Fig. (3) Comparison Between Numerical (Present Study) And Experimental [Koraim (2005)] Transmission Coefficients For The Semi-Immersed Model When B/H = 1.5 For Different Wave Conditions 
NUMERICAL SIMULATION OF PERMEABLE CAISSONS BREAKWATERS
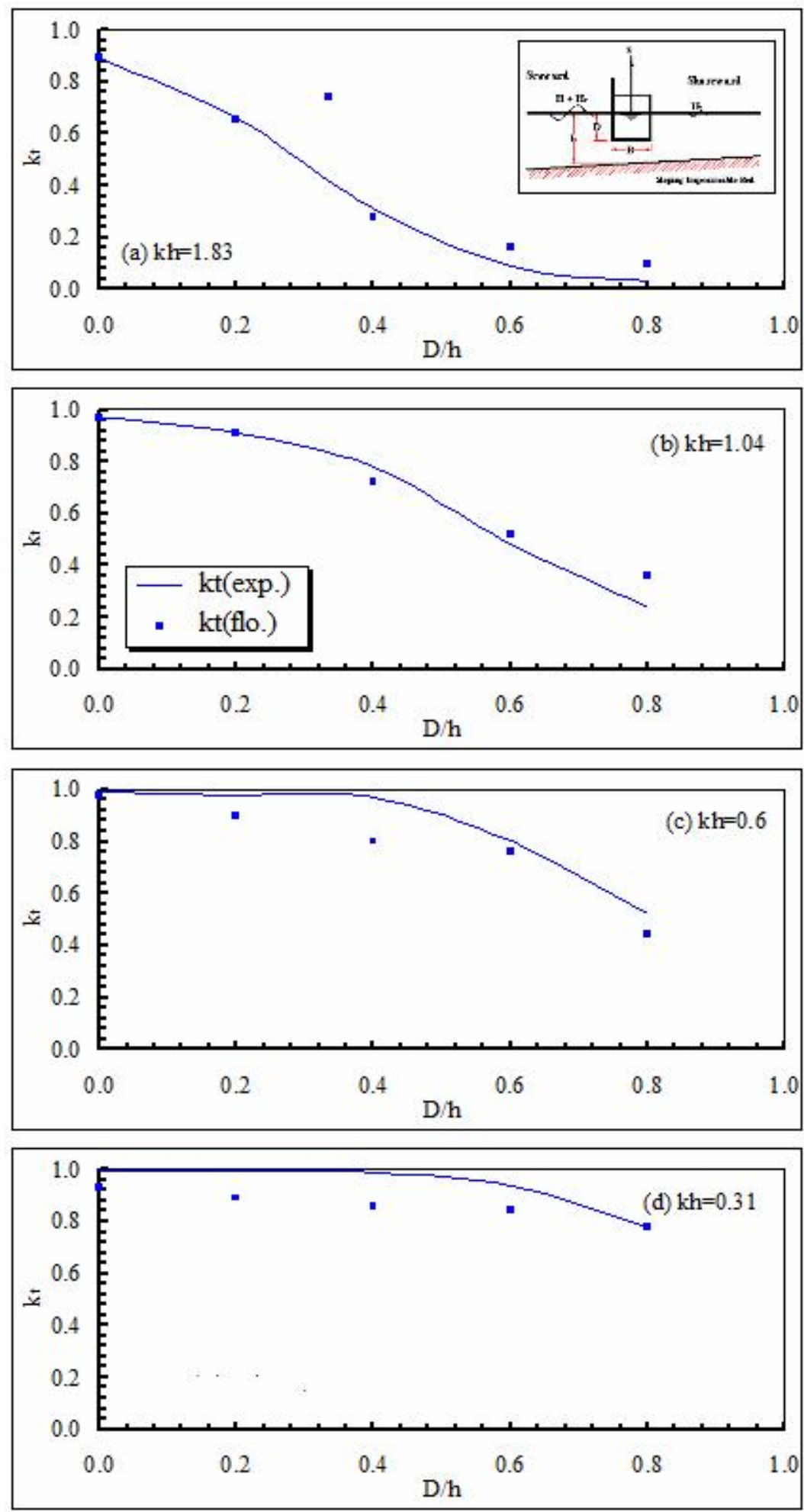

Fig. (4) Comparison Between Numerical (Present Study) And Experimental [Koraim (2005)] Transmission Coefficients For The Semi-Immersed Model When B/H = 0.5 For Different Wave Conditions 

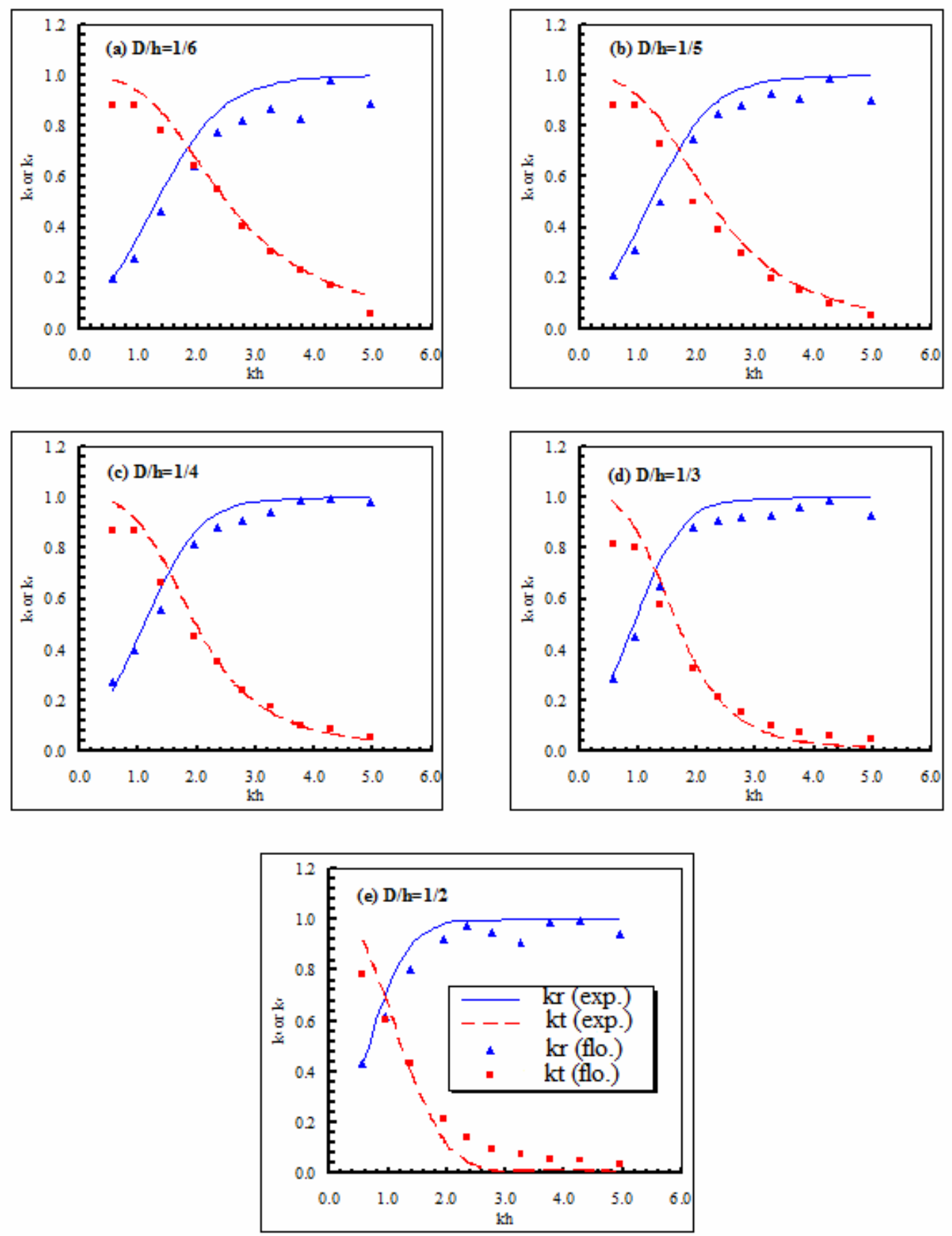

Fig. (5) The Comparison Between Numerical (Present Study) And Experimental [Koraim (2005) ] Transmission And Reflection Coefficients For Fixed Floating Breakwater Of $\mathbf{B} / \mathbf{H}=0.5$ For Different Relative Draft Ratios

\subsection{Comparison of the present numerical model with previous studies and affect draft.}

Figure (6) presents a comparison among the present numerical model [FLOW-3D] and experimental results and other empirical and experimental results presented in different studies, 
for a relative breakwater width $(\mathrm{B} / \mathrm{h})$ of 0.5 when $\mathrm{D} / \mathrm{h}=0.25$ and 0.5 . The figure also shows a reasonable agreement between the author's present numerical and the previous experimental results [Koraim (2005)]. Additionally, the figure shows that the author's numerical model introduced lower values of transmission coefficients than other empirical equations presented by other authors, for the same studied case. The numerical Results of the Caisson Breakwater as in figure (7).The relationship between the numerical transmission and reflection coefficients $(\mathrm{kt}$ and $\mathrm{kr})$, [FLOW-3D], and the dimensionless wave number (kh), is shown in Figure (7), for different D / h values, when the relative breakwater width (B / h) is equal to 1.0. Figure (7a) shows that the numerical transmission coefficient (kt) decreases with increasing $\mathrm{kh}$ and $\mathrm{D} / \mathrm{h}$. On the other hand, Figure (7b) shows that the numerical reflection coefficient (kr) increases with increasing kh and D / h.
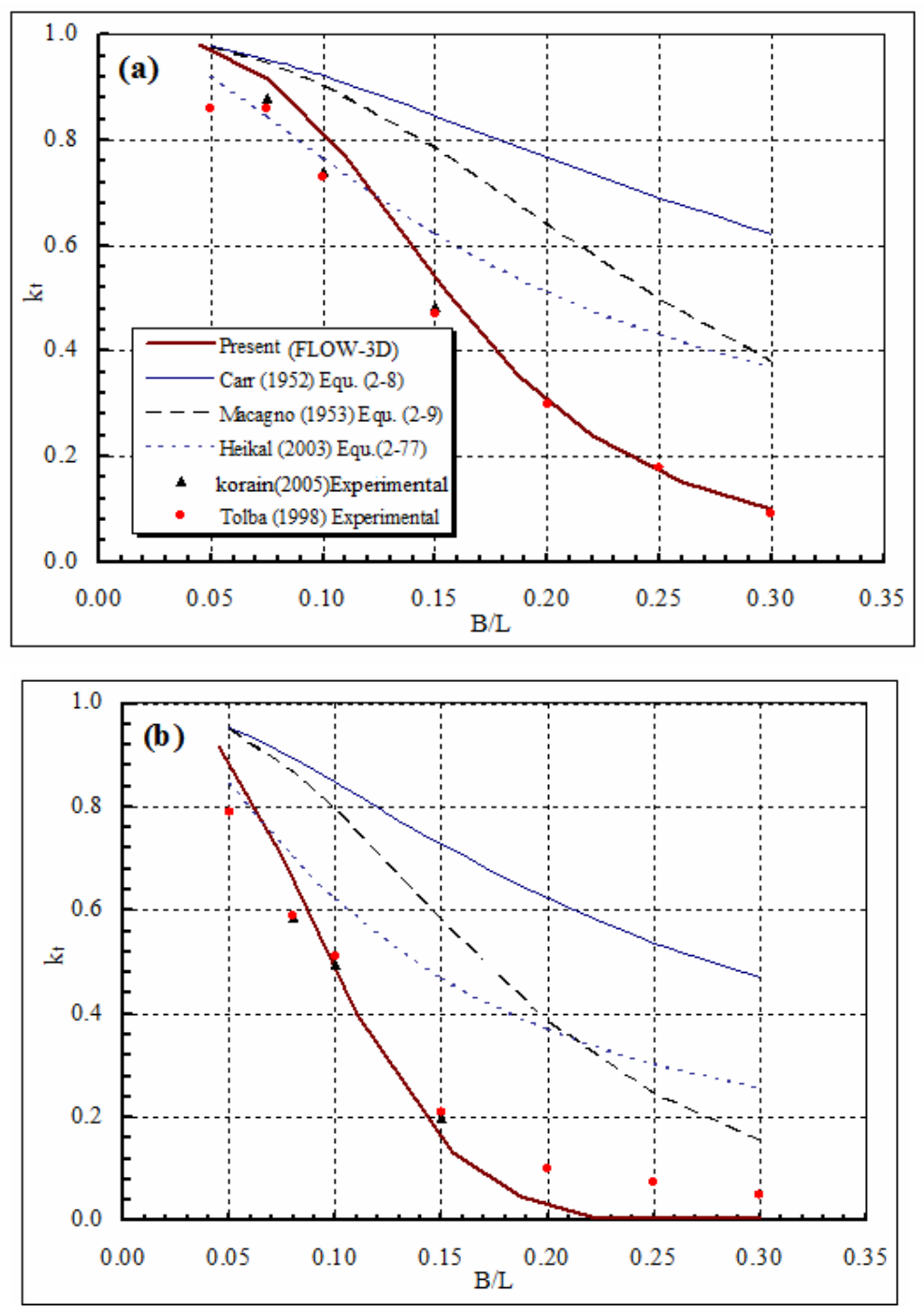

Fig. (6) Comparison Among Transmission Coefficients Obtained By Different Studies When $B / H=0.5$ And $S=$ 0\% For: (A) $\mathrm{D} / \mathrm{H}=\mathbf{0 . 2 5} \quad$ (B) $\mathrm{D} / \mathrm{H}=\mathbf{0 . 5 0}$

4.3 Effect of the slope of the seabed on the hydrodynamic performance of the barrier Figure (8) presents the relation between the relative breakwater width $(\mathrm{B} / \mathrm{h})$ and the transmission coefficient (kt), for different relative breakwater drafts $(\mathrm{D} / \mathrm{h})$, and seabed slopes $(\mathrm{sb})$, for a 
dimensionless wave number $(\mathrm{kh})$ of 1.88 . The figure shows that for all bed slopes, the transmission coefficient (kt) decreases as the relative breakwater width $(\mathrm{B} / \mathrm{h})$ increases. The transmission coefficient (kt) decreases with increasing sea-floor slope. For short waves, the wave barrier is very effective in reducing the energy of the transverse wave, regardless of structural arrangements and sea floor conditions. This may be due to increased friction between the surface of the breakwater and the transmitted waves, causing further loss of wave energy through the barrier. In addition, this condition may be due to the vortices shedding at the bottom tip of the barrier during transmission. The figure also shows clearly that the transmission coefficient decreases as the relative breakwater draft (D / h) increases. This behavior may be attributed to reduce wave energy transferred due to decreasing water area path through.
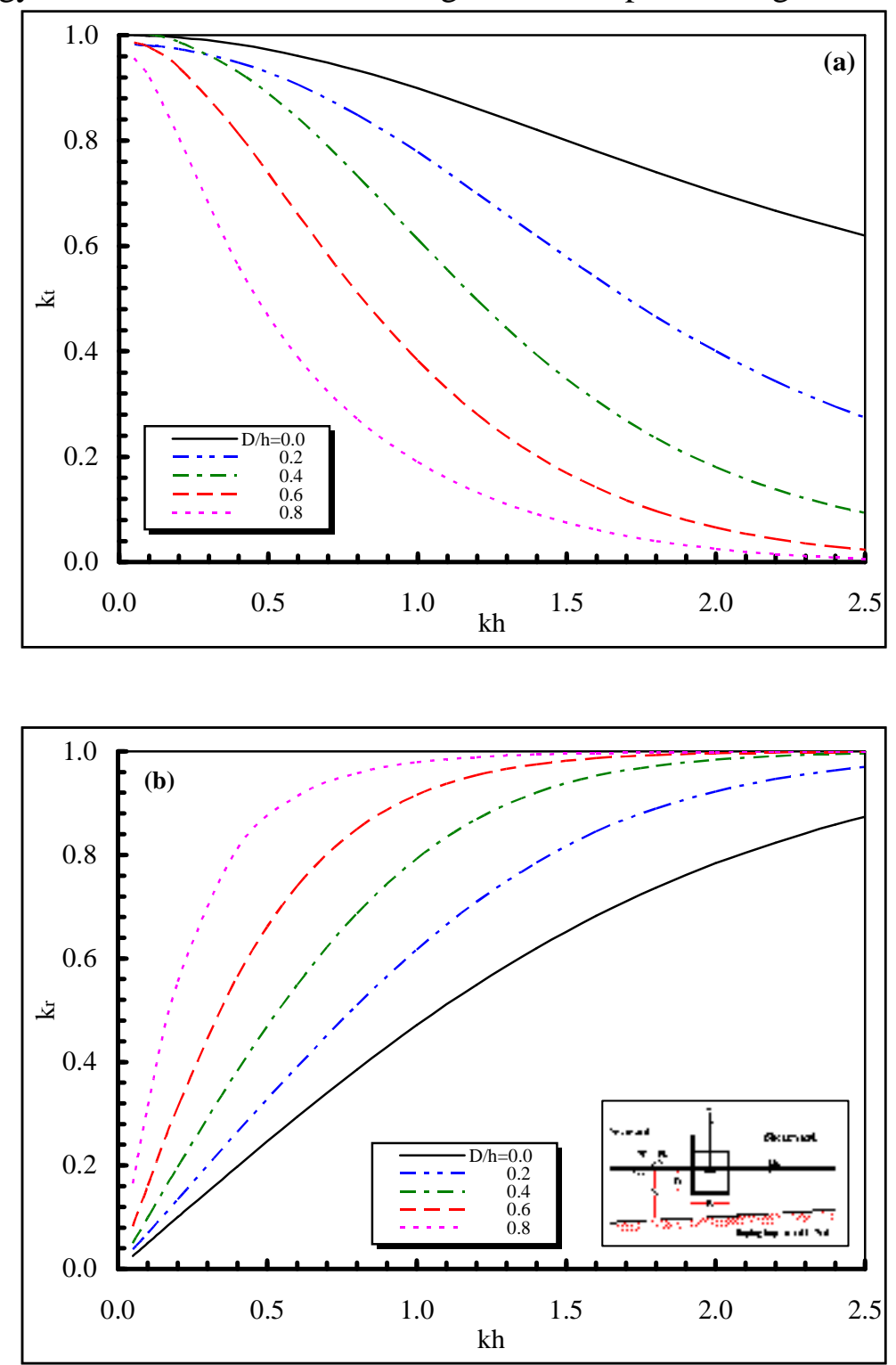

Fig. (7) The Relation Between The Numerical Transmission And Reflection Coefficients (Kt And Kr ) [ FLOW-3D ] And The Dimensionless Wave Number $(\mathrm{Kh})$ When $\mathrm{B} / \mathrm{H}=1.0$ 
NUMERICAL SIMULATION OF PERMEABLE CAISSONS BREAKWATERS
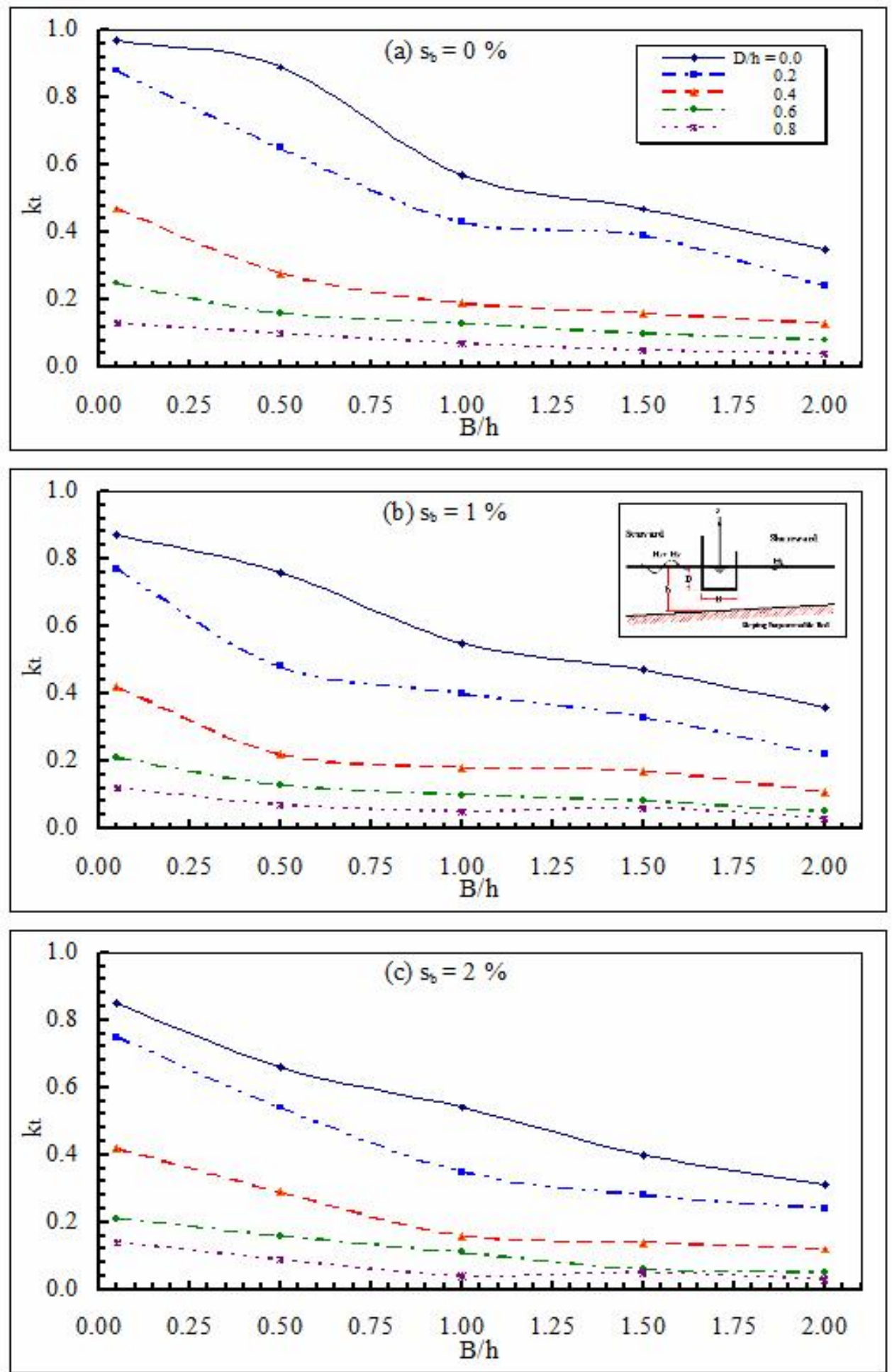

Fig. (8) The Relation Between The Transmission Coefficient (Kt) And The Relative Breakwater Width (B/H) For Different Breakwater Draft $(\mathrm{D} / \mathrm{H})$ And Seabed Slopes $(\mathrm{Sb})$ When $\mathrm{Kh}=1.88$ 


\section{DISTRIBUTION OF HYDRODYNAMIC PRESSURE, VORTEXES AND VELOCITIES OF WAVES AROUND PROPOSED BARRIERS}

Figure (9) shows the hydrodynamic pressure caused by the waves. The hydrodynamic pressure value is great in front of the breakwater and at the crest of the wave. The horizontal axis is $(\mathrm{x}) \mathrm{cm}$ and the vertical axis is $(\mathrm{z}) \mathrm{cm}$. The unit of hydrodynamic pressure gauge is Pascal Figures (10) shows the wave vortices around proposed breakwaters at different wave periods, Figures shows that when the wave period is shorter, the wave length is also short, leading to increased vortices around the barrier. So vortices in figures $(10 \mathrm{~b})$ for wave period $=1.0 \mathrm{sec}$. is bigger than figures (10a) for wave period $=1.2 \mathrm{sec}$. it's mean that the wave vortices increase around the barrier as the wave period decreases. The horizontal axis is $(\mathrm{x}) \mathrm{cm}$ and the vertical axis is $(\mathrm{z}) \mathrm{cm}$. vortex measurement unit (cm/s) Figures (11) shows the wave velocities around proposed breakwaters at different wave periods, Figures shows that when the wave period is shorter, the wave length is also short, leading to increased velocities around the barrier. The horizontal axis is $(\mathrm{x}) \mathrm{cm}$ and the vertical axis is $(\mathrm{z}) \mathrm{cm}$. Unit of measurement of wave velocity $(\mathrm{cm} / \mathrm{s})$. So velocities in figures (11c) for wave period $=0.9 \mathrm{sec}$. is big, velocities in figures $(11 \mathrm{~b})$ for wave period $=1.0 \mathrm{sec}$. is medium and velocities in figures (11a) for wave period $=1.2 \mathrm{sec}$. is small, it's mean that the wave velocities increase around the barrier as the wave period decreases.

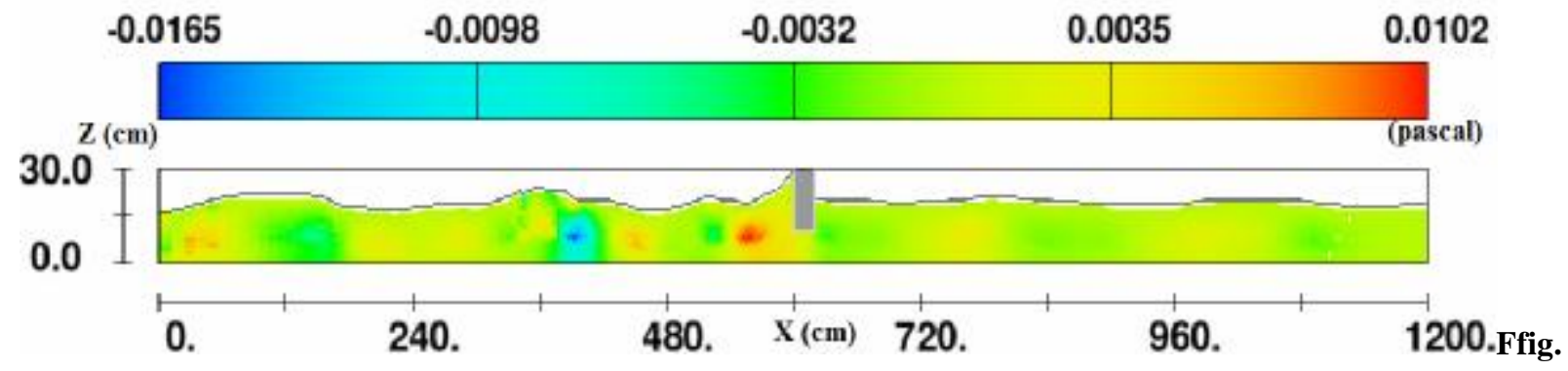

(9) Shows the hydrodynamic pressure around the breakwater.

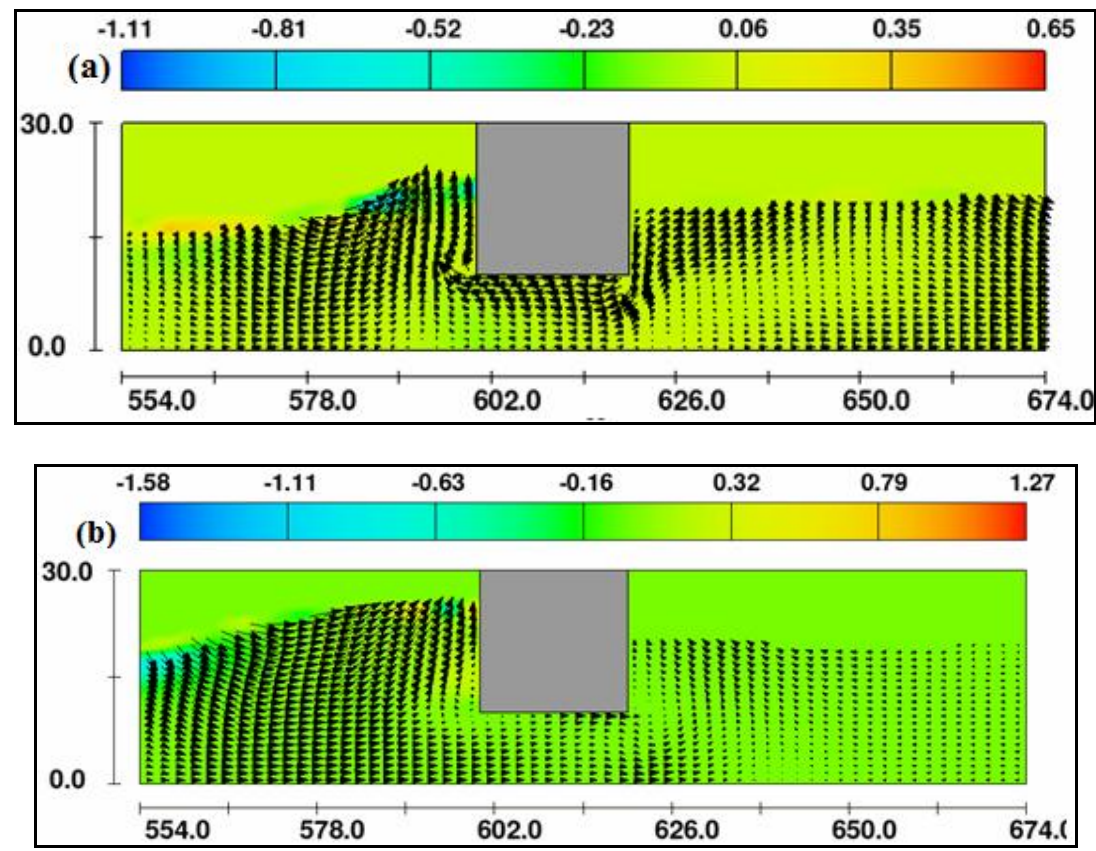

Fig. (10) Distribution Of Wave Vortices Around Proposed Breakwater Without Piles For Different Wave Period (T) At (A) T=1.2 Sec. And (B) T=1.0 Sec. 

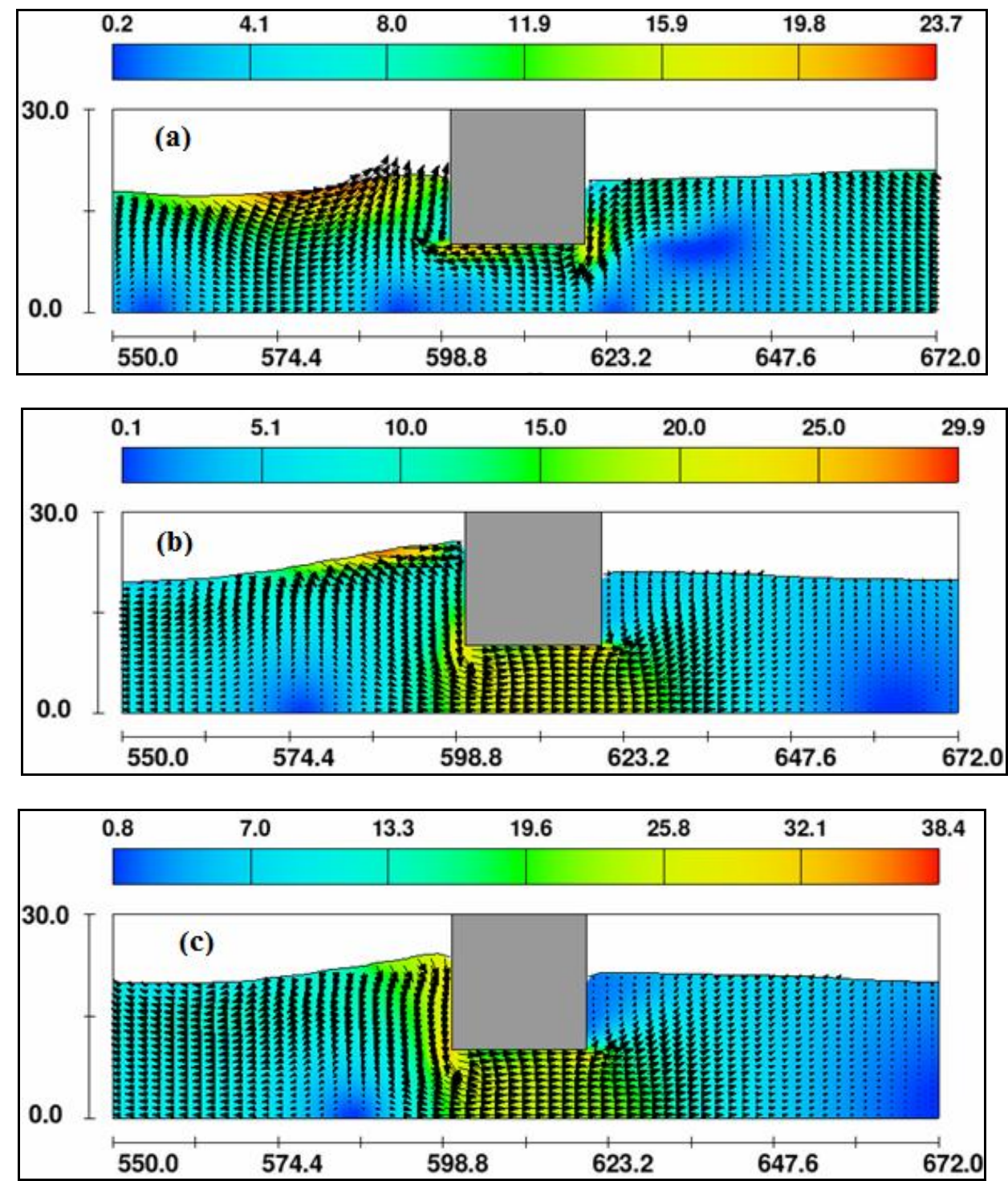

Fig. (11) Distribution Of Wave Velocities Around Proposed Breakwater Without Piles For Different Wave

\section{CONCLUSIONS}

Period (T) At (A) T=1.2 Sec., (B) T=1.0 Sec. And (C) $T=0.9$ Sec.

The current research is summarized in the following points.

1. This research shows a great agreement between the results of the present numerical model and the previous laboratory model.

2. The transmission coefficient (kt) decreases as the relative breakwater width $(\mathrm{B} / \mathrm{h})$ increases, the transmission coefficient (kt) decreases as the relative breakwater draft (D / h) increases.

3. At all bed slopes, the transmission coefficient $(\mathrm{kt})$ decreases as the relative breakwater width $(\mathrm{B} / \mathrm{h})$ increases.

4. The proposed breakwater reduces the velocity of the waves behind and also disperses the waves through it.

5. The hydrodynamic pressure value is great in front of the breakwater and at the crest of the wave. 


\section{REFERENCES}

1. Macagno. E. O., (1953) "Fluid Mechanics Experimental Study of the Effects of the Passage of a Wave Beneath an Obstacle" Proceedings of the Academic des Sciences, Paris, France

2. Stoker, J. J. (1957) "Water Waves, the Mathematical Theory with Applications" Copied by Inter Science Publishers, New York.

3. Carver, R. D. (1979) "Floating Breakwater Wave-Attenuation Tests for East Bay Marina, Olympia Harbor, Washington; Hydraulic Model Investigation" Technical Report HL7913, U. S. Army Engineer Waterways Experiment Station, CE, Vicksburg, Mississippi, July.

4. Reddy, M.S. and Neelamanit, S. (1992) "Wave Transmission and Reflection Characteristics of a Partially Immersed Rigid Vertical Barrier" J. of Ocean Engineering, Vol. 19, No. 3.

5. Ito, Y. and Chiba, S. (1972) "An Approximate Theory of Floating Breakwaters" Report of the Port and Harbor Research Institute, Ministry of Transportation, Japan, Vol. 11, No. 2.

6. Adee, B. H. and Martin, W. (1974) "Theoretical Analysis of Floating Breakwater Performance" Proc. Of the Floating Breakwater Conf., Univ. of Rhode Island, Kingston, Rhode Island.

7. Sutko, A. A. and Haden, E. L. (1974) "The Effect of Surge, Heave and Pitch on the Performance of a Floating Breakwater" Proc. Of the Floating Breakwater Conf., Newport, Rhode Island.

8. Fugazza, M. and Natale, L. (1988) "Energy Losses and Floating Breakwater Response" J. Waterway, Port, Coastal and Ocean Eng., ASCE, Vol. 114, No

9. Tolba, E.R. (1998) "Behavior of floating breakwater under wave action" Ph. D. Thesis, Suez Canal University, Egypt.

10. Heikal, E.M. (2004) "Local Seabed Scour at Vertical Piles Superimposed by a Semiimmersed Body" J. of Scientific Bulletin, Eng. Faculty, Ain-Shams Univ., Vol. 39, No. 2.

11. Ioanna Diamantoulaki and Demos C. A. (2009) "Three-dimensional Formulation of Multiple Pile-restrained Floating Breakwaters Connected by Hinges" the Nineteenth International Offshore and Polar Engineering Conference, Osaka, Japan, June 21-26, 2009.

12. Huan-Ya Wang and Zhao-Chen Sun (2010) "Experimental study on the influence of geometrical configuration of porous floating breakwater on performance" J. of Marine Science and Technology, Vol. 18, No. 4, pp. 574-579.

13. Rageh, O., Koraim, A. (2010). "Hydrodynamic efficiency of partially immersed caissons supported on piles". J. Ocean Eng. 36 (14), 1112-1118

14. Koraim, A.S., (2005). Suggested Model for the Protection of Shores and Marina. Zagazig University, Zagazig, Egypt (A Thesis of Ph.D. in Civil Engineering).

15. Hany Ahmed,(2014)"proposing and investigating the efficiency of an innovative economic breakwater" Al-Azhar Engineering twelfth International Conference December $24-26,2014$

16. Mohamed Ibrahim (2018). "Linear Wave Interaction with Permeable Breakwaters". A Thesis Submitted for Partial Fulfillment of Doctor of Philosophy Degree in Civil Eng., alAzhar University. 